\title{
Correction to: Classifying MOOC forum posts using corpora semantic similarities: a study on transferability across different courses
}

\author{
Anastasios Ntourmas ${ }^{1}$ (D) $\cdot$ Sophia Daskalaki ${ }^{1} \cdot$ Yannis Dimitriadis $^{2} \cdot$ Nikolaos Avouris $^{1}$
}

Published online: 22 April 2021

(C) Springer-Verlag London Ltd., part of Springer Nature 2021

\section{Correction to: Neural Computing and Applications} https://doi.org/10.1007/s00521-021-05750-z

The article Classifying MOOC forum posts using corpora semantic similarities: a study on transferability across different courses, written by Anastasios Ntourmas - Sophia Daskalaki · Yannis Dimitriadis · Nikolaos Avouris, was originally published electronically on the publisher's Internet portal (currently SpringerLink) on [04 February
2021] with Open Access under a "Creative Commons Attribution (CC BY) license 4.0".

With the author's/authors' decision to cancel Open Access the copyright of the article changed on 5th, March, 2021 to (C) Springer-Verlag London Ltd., part of Springer Nature 2021 with all rights reserved.

Publisher's Note Springer Nature remains neutral with regard to jurisdictional claims in published maps and institutional affiliations.

The original article can be found online at https:// doi.org/10.1007/s00521-021-05750-z.

Anastasios Ntourmas

a.ntourmas@upnet.gr

Sophia Daskalaki

sdask@upatras.gr

Yannis Dimitriadis

yannis@tel.uva.es

Nikolaos Avouris

avouris@upatras.gr

1 Electrical and Computer Engineering Department, University of Patras, Patras, Greece

2 School of Telecommunications Engineering, Universidad de Valladolid, Valladolid, Spain 